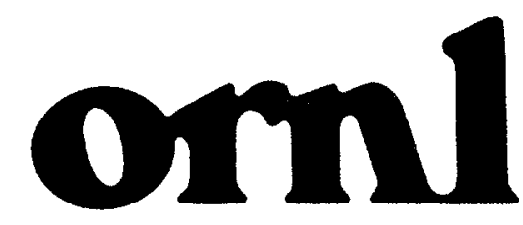

OAK RIDGE NATIONAL LABORATORY

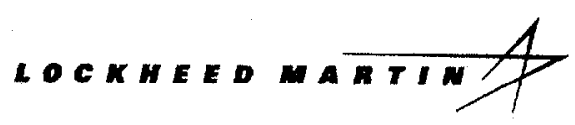

\section{Measurement of Dynamic Strain on a Mercury Target Vessel During 800-MeV Proton Thermal Shock}

M. R. Cates, S. W. Allison, J. B. Andriulli, D. D. Earl, J. R. Haines, F. X. Morrissey, C. C. Tsai

Oak Ridge National Laboratory

Oak Ridge, Tennessee 37831-8058 USA

S. Wender

Los Alamos National Laboratory

Los Alamos, New Mexico 87545 USA

Prepared by the

Oak Ridge National Laboratory

Oak Ridge, Tennessee 37831-8047

Managed by

LOCKHEED MARTIN ENERGY

RESEARCH CORP.

for the

U.S. DEPARTMENT OF ENERGY

under contract DE-AC05-96OR22464. 



\section{MEASUREMENT OF DYNAMIC STRAIN ON A MERCURY TARGET VESSEL DURING 800-MeV PROTON THERMAL SHOCK TESTS}

M. R. Cates

Oak Ridge National Laboratory

Oak Ridge TN 37831-8058

(423) $574-8056$

D. D. Earl

Oak Ridge National Laboratory

Oak Ridge TN 37831-8058

(423) 576-0869

C. C. Tsai

Oak Ridge National Laboratory

Oak Ridge TN 37831-8071

(423) 574-1124
S. W. Allison

Oak Ridge National Laboratory

Oak Ridge TN 37831-8058

(423) 576-2725

J. R. Haines

Oak Ridge National Laboratory

SNS Project Office Rm 171

Oak Ridge, TN 37831-8218

(423) 574-0966

\author{
F. X. Morrissey \\ Oak Ridge National Laboratory \\ Oak Ridge TN 37831-8058 \\ (423) 241-3118
}

J. B. Andriulli

Oak Ridge National Laboratory

Oak Ridge TN 37831-8088

(423) 576-0424

Stephen Wender

Los Alamos National Laboratory LANSCE

Los Alamos NM 87545

(505) 667-1344

\section{ABSTRACT}

A mercury target vessel, designed to simulate some aspects of the eventual target design for the proposed Spallation Neutron Source (SNS) to be built in Oak Ridge by the Department of Energy, was used in a test at the Los Alamos Neutron Science Center (LANSCE) to study the strain induced from thermal shock of bombarding protons. In the SNS, intense thermal shock loads are expected to cause an enormous rate of temperature rise $\left(-10^{7} \mathrm{~K} / \mathrm{s}\right)$, with resulting pressure waves in the mercury that may lead to large stresses on the thin walls of the mercury target. To guide the mercury target design and to benchmark the computer design codes, transient strain was measured using fiber optic Fabry-Perot sensors. Twenty strain sensors were attached in various axial and transverse orientations to a cylindrical stainless steel target vessel containing mercury. The vessel was $10 \mathrm{~cm}$ in diameter, about $15 \mathrm{~cm}$ long, and with a $5-\mathrm{cm}$ radius hemispherical shell welded to the forward end. The test was done at the LANSCE Weapons Neutron Research (WNR) beam facility on 30-31 January 1999. The sensors were attached with gauge lengths of about two centimeters, and were located in pairs in most areas, for redundancy and facilitation of data analysis. The $800-\mathrm{MeV}$ proton deposition of $0.5-2.3 \times 10^{13}$ over a full-width at half maximum beam size of $\sim 25 \mathrm{~mm}$, produced axial strains peaking at a few microstrains, with transverse (hoop) strains more than an order of magnitude higher. We describe the experiments, including the sensors and measurement configuration, and discuss the strain data analysis.

\section{INTRODUCTION}

The Spallation Neutron Source (SNS) being designed and built by the United States Department of Energy will use a high-intensity target comprised of liquid mercury contained within a stainless steel vessel. Mercury was chosen both for its high efficiency as a spallation source and because it is a liquid metal at ambient temperatures. The liquid ambient phase will not show permanent damage from proton bombardment, and can be cooled by flowing through appropriate heat exchangers. The major concern associated with using mercury as a target material is the potential deleterious effects of thermal shock on the target vessel. Sophisticated computer codes are used to model the target configuration, and a series of measurements is being undertaken to benchmark or validate these codes. With the beam per pulse for SNS of about $2 \times 10^{14}$ protons at an energy of $1 \mathrm{GeV}$, the near-instantaneous temperature rise per unit time is predicted to be around $10^{7} \mathrm{~K} / \mathrm{s}^{1,2}$ For pulse widths on the order of microseconds, the anticipated temperature rise per pulse may be several degrees. For the SNS pulse repetition rate of $60 \mathrm{~Hz}$, handling the time- 
averaged power is relatively straightforward, whereas the repeated long-term effects of thermal shock remain a concern.

A measure of the effect of thermal shock is the strain induced in the vessel by the proton bombardment. This project has focused on developing the measurement system necessary for monitoring strain from thermal shock, and testing the system in tests at the Los Alamos Neutron Science Center (LANSCE) using a mercury target vessel. The tests took place on January 30-31,1999.

\section{MERCURY CONTAINMENT TEST VESSEL}

Figure 1 shows a drawing of the mercury vessel used in the LANSCE test. The vessel was $10 \mathrm{~cm}$ (4 inches) in diameter, about $15 \mathrm{~cm}$ (6 inches) long, and with a $5-\mathrm{cm}(2-$ inch) radius hemispherical shell welded to the forward end. It was made of stainless steel about $2 \mathrm{~mm}$ thick $(0.08$

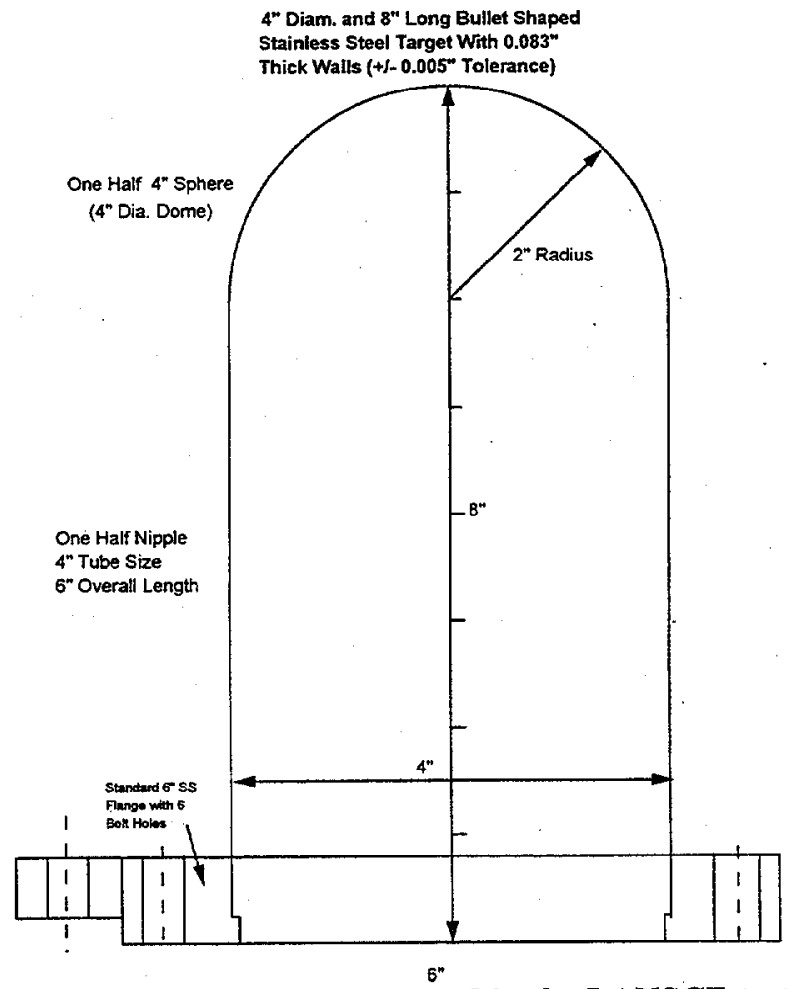

Figure 1. Mercury vessel used in the LANSCE test.

inch). This vessel was identified as Target $A$ for the test program because there was another test vessel also studied (identified as Target B). On the back flat surface of Target A were four ports for pressure and temperature sensors also used in the test. The vessel was leak tested after fabrication, then emptied of mercury for sensor installation and transport to Los Alamos. High purity mercury was added just prior to installation in the beam line of the
Weapons Neutron Research (WNR) test chamber, called the Blue Room. A secondary containment vessel, also made of stainless steel, was used during the test. This vessel was essentially a rectangular box on the outside of which were located reference marks for alignment. After the experiment ended on January 31, 1999, a small sample of mercury was removed for radioactive analysis, and the target vessel stored on site at LANSCE for possible later use.

\section{FABRY-PEROT STRAIN SENSORS}

Twenty strain sensors were attached in various axial and transverse orientations to the outside of Target $A$. The sensors were optical fiber Fabry-Perot interferometric designs with the high sensitivity (fractions of a microstrain) necessary for the SNS study. They were manufactured by F\&S, Inc., of Blacksburg, Virginia. A diagram of the Fabry-Perot strain sensors (FPSS) is shown in Figure 2.

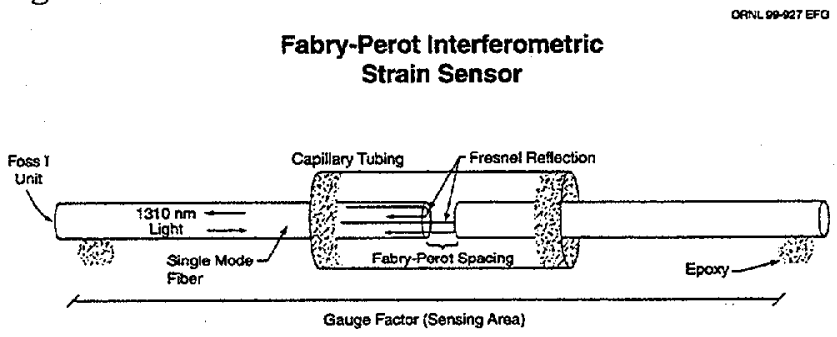

Figure 2. Fabry-Perot strain sensors.

The sensing mechanism is as follows. Light (1) from a semiconductor laser is propagated down a single-mode optical fiber, the light is (2) partially reflected from both the end of the propagation fiber and the front surface of a short co-linearly aligned multi-mode fiber a few tens of microns away. The two reflecting surfaces (3) are contained and maintained in alignment by a tiny capillary tube. Because of the relatively short coherence length of the laser, the mode dispersion in the extension of the short fiber prevents any other interference within the system from affecting the signal. As the spacing (4) between the fibers is changed, the return reflectance propagating back through the source fiber will experience interference. As the fiber spacing changes one-half wavelength of the laser emission, the return signal goes through one full interference fringe. The sensor is attached to the stainless steel surface by small droplets of epoxy, the distance between these droplets being the gauge length, $\mathrm{L}$. The amount of change in the fiber spacing corresponds to the expansion or contraction of the surface within that gauge length. The strain $S$ is thus expressed:

$$
S=\Delta X / L,
$$


where $\Delta \mathrm{X}$ is the displacement of the fiber spacing, and $\mathrm{L}$ the gauge length. Often $S$ is expressed in microstrain $\left(10^{-6}\right.$ strain), with $\Delta X$ in microns and $L$ in meters. For this type of sensor, the signal output goes through a series of interference fringes as displacement changes. The displacement $\mathrm{X}_{\mathrm{I}}$ of the sensor gap is determined by the expression:

$$
\mathrm{V}_{\mathrm{i}}=\sin \mathrm{X}_{\mathrm{i}}
$$

where $V_{i}$ is the signal that results from the displacement $X_{i}$. To find $\Delta X$ it is necessary to measure the difference between $X_{i}$ and the unstrained displacement $X_{0}$. This displacement $\Delta \mathrm{X}$, because of the light interference, is dependent on the wavelength $\lambda$ of the light source. The light source for the measurements here was a laser diode emitting at 1.310 microns. Because the sensor gap is traversed both across and back by the interfering light (see Figure 2), one full fringe (or sine wave cycle) corresponds to a fiber displacement of $1 / 2 \lambda$, or 0.655 micron. It is convenient to express $\Delta \mathrm{X}$ in terms of fractions of a fringe $\Delta \mathrm{F}$. The expression for strain can be written

$$
S=1000[\Delta F(\lambda / 2) / L],
$$

with $\mathrm{S}$ in micro-strain, $\mathrm{L}$ in $\mathrm{mm}$, and the laser wavelength $\lambda$ in microns. A 90-degree change in the fringe pattern ( $\triangle F$ of $1 / 4$ ) would represent a strain of 1.64 microstrain per millimeter of gauge length. The gauge lengths $(L)$ for the sensors used in this test were typically between 15 and 20 $\mathrm{mm}$; consequently, the amount of strain per quarter fringe was on the order of 10 microstrain.

The data become more complicated to analyze when the displacement exceeds a $\Delta \mathrm{F}$ of more than $1 / 4$ fringe. In those cases the interference signal is likely to go through maximum constructive or destructive phases (maximum or minimum output signal), often causing the output voltage amplitude to go through a reversal, departing a monotonic response to changing strain. The sine wave interference pattern is then expressed mathematically in different quadrants of the angle progressing from 0 to 360 degrees ( 0 to $2 \pi$ radians). The ambiguity introduced by the cyclic interference behavior is part of the well-known fringecounting problem of such systems, and cannot be accounted for without more information than is contained in the output signal for systems using a single wavelength of light. To help decipher this ambiguity, pairs of sensors were used. Despite these problems with interference fringes, the requirement of sensitivity and speed of response forced us into using sensors of this type. The only commercially available sensors that met our scheduling and cost constraints were single wavelength FPSS, with the unavoidable signal ambiguity. For the sensors available there were no commercial signal processing systems that werc fast enough to follow the expected strain response of the target vessel; consequently, we were required to analyze the output signals on the basis of calibration and reference information that we had at our disposal. We knew from the beginning that some signals might be too ambiguous to unfold with a high degree of confidence, but that others would probably be much more reliably interpreted. That expectation turned out to be the case for the experimental data.

\section{MEASUREMENT SYSTEM}

The components in the measurement system were: (a) individual sensors, attached to the target vessel at various locations, connected by optical fiber pigtails to 40 -meter fibers (b) that terminated at an optical patch panel outside the WNR Blue Room. Up to six channels (six fibers) could be connected from the patch panel to terminals (c) on a Fiber Optic Sensor System (FOSS) module that provided the laser input and an analog voltage output from the return light signal. The FOSS outputs were routed into digital oscilloscopes (d), and through appropriate interfaces into personal computers (e), where the signal was stored and some preliminary analysis performed. Figure 3 is a photograph of Target $\mathrm{A}$ during the sensor application stage, showing attached sensors. The calibration done for each attached sensor was to measure the peak-to-peak fringe signal voltage by forcing the sensor (usually by heating) to negotiate several fringes. Unfortunately, the sensor arrangement in the LANSCE test was changed enough during the course of the various runs that the optical connections could not be expected to exactly repeat the peak-to-peak response of a particular sensor. It was true; however, that for the sensors, which actually went through a set of peak maxima and minima, the observed peak-to-peak was typically within about $10 \%$ of the calibration value.

Figure 4 shows two photographs of the measurement arrangement in the hallway outside the WNR Blue Room at LANSCE. The scope data could be observed directly after each beam pulse, and was sent automatically into a storage and analysis routine. ${ }^{3}$ The routine provided a linear correlation of the strain signal and folded in the various calibration parameters, giving useful estimates of the strain for conditions where the signal displacement was less than the peak-to-peak fringe signal. This same routine had been used in a previous accelerator test. The software also stored a video image of the proton beam profile captured by a frame-grabber triggered by the event. 


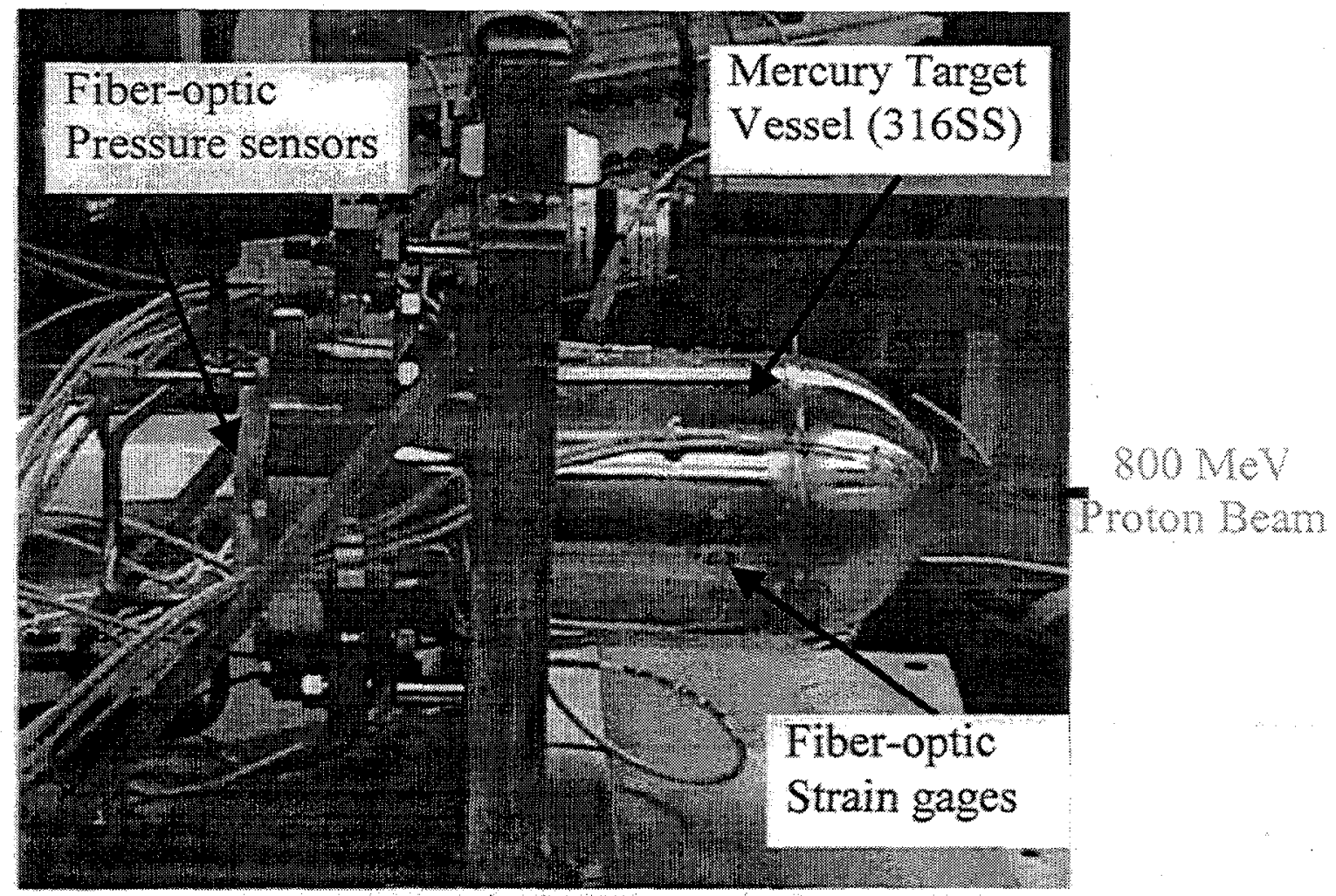

Figure 3. Target A during the sensor application stage.
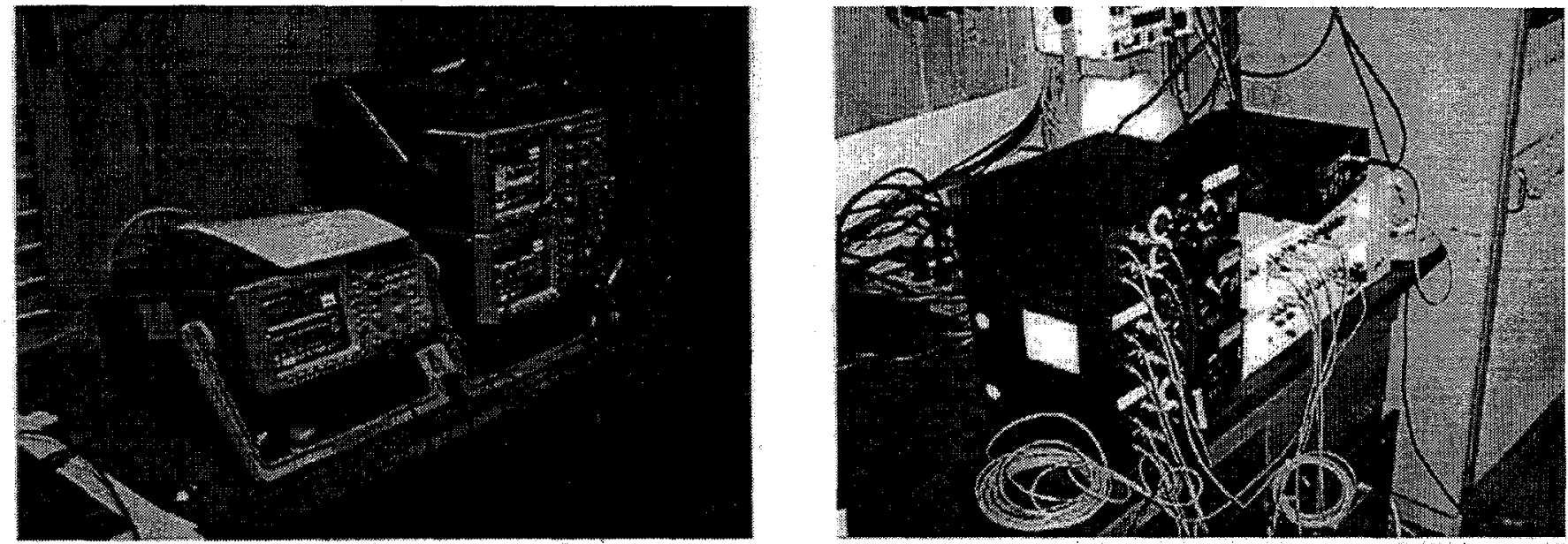

Figure 4. Measurement arrangement in the hallway outside the WNR Blue Room at LANSCE.

\section{DATA AND ANALYSIS}

Target A was configured with 20 sensors, their locations diagrammed in Figure 5. The positions are listed in terms of the axial direction back from the front of the target, and angular position in viewing the target from the front. This target was used in 35 beam pulses, labeled in order TA1 through TA35. The proton flux per pulse was measured and recorded for use in normalization and interpretation of the data. Table 1 summarizes some of the relevant information from the test and the sensors, including an estimate of the maximum voltage output swing of the strain data. This output peak-to-peak, compared with the calibration peak-to-peak voltage from the sensor, gave 
an indication of whether the strain had displaced the sensor gap more than a quarter fringe. Typically, the sensors near the target front and the sensors configured to measure the transverse (hoop) strain exceeded this quarter fringe reference. Those farther back and axially aligned generally did not.

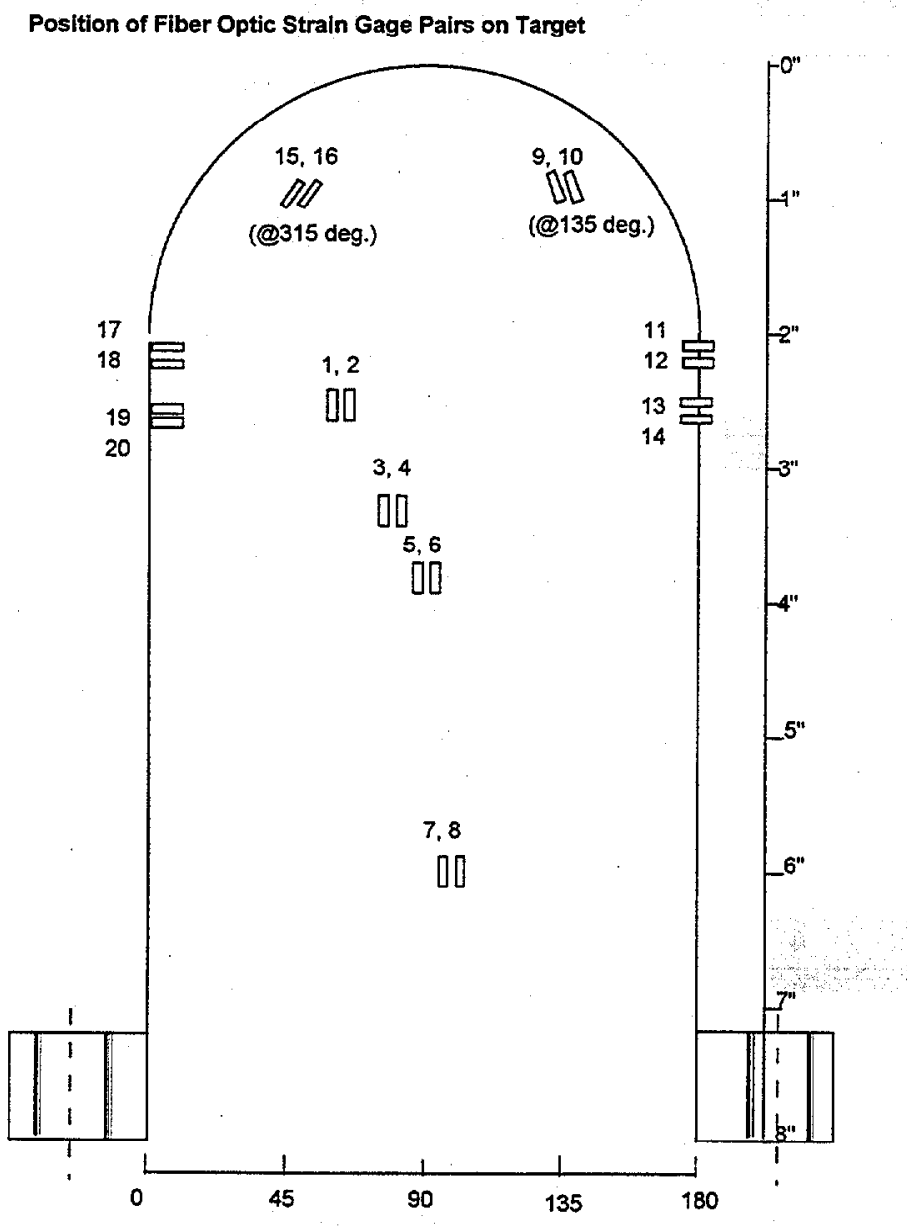

Figure 5. Target $\dot{A}$ sensor configuration.

To extract strain from the voltage outputs of the sensors, it is necessary to know three basic things: (1) the sensitivity, extracted from the peak-to-peak calibration for the sensor, (2) the beginning (zero strain) phase of the sensor, and (3) the location in time of any turn-around of the signal after negotiating some number (or fraction) of fringes. For the sensitivity, each set of data has a good estimate from the pre-test calibration, although signal amplitude variations can result from additional optical connections that occur in the test. In cases where the actual test data delineate the full extent of the detector output, the observed peak-to-peak swing defines the sensitivity. In those cases where the peak-to-peak signal swing is produced, the reference phase is also directly available, because the starting (zero strain) voltage will lie some- where between the peak-to-peak limits, and can therefore be seen at a certain phase (angle) of the sine wave pattern of the fringes. If we make the further assumption that the first large excursion of the signal data represents a positive (expansion) strain, then the starting voltage can be further identified as in a particular one of the four quadrants of the sine function. There, the inverse sine can be directly extracted as a function of time to yield displacement, thence strain.

Analysis of all of the LANCE Target A strain data is not yet complete, but some important aspects of the measurements can be reported. In this paper, we emphasize two areas of the measurement: (1) study of repeated data sets and (2) study of data sets with scaled proton pulse power. This study involves sensors 5 and 6 (see Figure 5), configured for axial strain measurement not far back from the interface between the front hemisphere and the cylindrical body of the target. Note the list in Table 1, where the pulses from TA15 through TA31 were done with sensors 5 and 6 in the same configuration, with optical connections undisturbed. TA15 through TA19 were at approximately the same proton power, with later pulses reduced to half, then quarter power. For all these data, the sensors went through something less than a quarter fringe of deflection, and had zero strain voltage levels that were somewhere close to the middle of the peak-to-peak range of their calibrations. The evidence for this latter condition is the fact that all the signal shapes looked very similar, especially for about the first millisecond, indicating that the starting phases were not near either the maximum or minimum turnaround points of the fringes. This is illustrated in Figure 6, which shows representative data signals from pulses at full power, half power and quarter power.

The fact that the peak voltage deflection in the signals shown in Figure 6 is never more than about half the peakto-peak calibration numbers for the particular sensor is an indication that the signals can be compared linearly with some degree of confidence. The logic is that for small deflections the value of the angle and its sine remain similar to each other. Similarly, since the deflections were relatively small, it was not necessary to consider the possibility that the signal reached the inflection points at the maximum or minimum voltage, i.e., the response does not depart from monotonic. Noting the peak signal deflections for these three decreasing beam powers gives values that are reasonably scaled to the relative power of the proton pulses. 
Table 1. Strain sensors used for January' 99 thermal shock measurements at LANSCE

\begin{tabular}{|c|cc|cc|c|}
\hline & \multicolumn{5}{|c|}{ Sensor number and peak-peak voltage per fringe } \\
\cline { 2 - 6 } Event No. & $\begin{array}{r}\text { Processing unit } \\
\text { peak-peak } \\
\text { (-A) }\end{array}$ & $\begin{array}{c}\text { Processing unit } \\
\mathbf{1 - B}\end{array}$ & $\begin{array}{c}\text { peak-peak } \\
\text { (V) }\end{array}$ & $\begin{array}{c}\text { Protons } \\
\text { per pulse }\end{array}$ \\
\hline TA-1 & $\mathbf{5}$ & 3.7 & $\mathbf{6}$ & 4.2 & $9.74 \mathrm{E}+12$ \\
\hline TA-2 & $\mathbf{5}$ & 3.7 & $\mathbf{6}$ & 4.2 & $1.01 \mathrm{E}+13$ \\
\hline TA-3 & $\mathbf{5}$ & 3.7 & $\mathbf{6}$ & 4.2 & $1.16 \mathrm{E}+13$ \\
\hline TA-4 & $\mathbf{5}$ & 3.7 & $\mathbf{6}$ & 4.2 & $1.13 \mathrm{E}+13$ \\
\hline TA-5 & $\mathbf{2}$ & 3.5 & 7 & 3.1 & $1.34 \mathrm{E}+13$ \\
\hline TA-6 & $\mathbf{2}$ & 3.5 & 7 & 3.1 & $1.40 \mathrm{E}+13$ \\
\hline TA-7 & $\mathbf{2}$ & 3.5 & 7 & 3.1 & $1.62 \mathrm{E}+13$ \\
\hline TA-8 & $\mathbf{1 9}$ & 3.5 & $\mathbf{2 0}$ & 3.9 & $2.08 \mathrm{E}+13$ \\
\hline TA-9 & $\mathbf{1 9}$ & 3.5 & $\mathbf{2 0}$ & 3.9 & $2.24 \mathrm{E}+13$ \\
\hline TA-10 & $\mathbf{3}$ & 1.5 & $\mathbf{9}$ & 2.4 & $2.29 \mathrm{E}+13$ \\
\hline TA-11 & $\mathbf{3}$ & 1.5 & $\mathbf{9}$ & 2.4 & $2.30 \mathrm{E}+13$ \\
\hline TA-12 & $\mathbf{2 1}$ & 0.7 & $\mathbf{2 2}$ & 7.5 & $2.29 \mathrm{E}+13$ \\
\hline TA-13 & $\mathbf{2 1}$ & 0.7 & $\mathbf{2 2}$ & 7.5 & $2.28 \mathrm{E}+13$ \\
\hline TA-14 & $\mathbf{2 1}$ & 0.7 & $\mathbf{2 2}$ & 7.5 & $2.28 \mathrm{E}+13$ \\
\hline TA-15 & $\mathbf{5}$ & 3.7 & $\mathbf{6}$ & 4.2 & $2.20 \mathrm{E}+13$ \\
\hline TA-16 & $\mathbf{5}$ & 3.7 & $\mathbf{6}$ & 4.2 & $2.19 \mathrm{E}+13$ \\
\hline TA-17 & $\mathbf{5}$ & 3.7 & $\mathbf{6}$ & 4.2 & $2.17 \mathrm{E}+13$ \\
\hline TA-18 & $\mathbf{5}$ & 3.7 & $\mathbf{6}$ & 4.2 & $2.23 \mathrm{E}+13$ \\
\hline TA-19 & $\mathbf{5}$ & 3.7 & $\mathbf{6}$ & 4.2 & $2.25 \mathrm{E}+13$ \\
\hline TA-20 & $\mathbf{5}$ & 3.7 & $\mathbf{6}$ & 4.2 & $2.23 \mathrm{E}+13$ \\
\hline TA-21 & $\mathbf{5}$ & 3.7 & $\mathbf{6}$ & 4.2 & $1.15 \mathrm{E}+13$ \\
\hline TA-22 & $\mathbf{5}$ & 3.7 & $\mathbf{6}$ & 4.2 & $1.14 \mathrm{E}+13$ \\
\hline TA-23 & $\mathbf{5}$ & 3.7 & $\mathbf{6}$ & 4.2 & $5.39 \mathrm{E}+12$ \\
\hline TA-24 & $\mathbf{5}$ & 3.7 & $\mathbf{6}$ & 4.2 & $5.34 \mathrm{E}+12$ \\
\hline TA-25 & $\mathbf{5}$ & 3.7 & $\mathbf{6}$ & 4.2 & $5.52 \mathrm{E}+12$ \\
\hline TA-26 & $\mathbf{5}$ & 3.7 & $\mathbf{6}$ & 4.2 & $5.54 \mathrm{E}+12$ \\
\hline TA-27 & $\mathbf{5}$ & 3.7 & $\mathbf{6}$ & 4.2 & $5.64 \mathrm{E}+12$ \\
\hline TA-28 & $\mathbf{5}$ & 3.7 & $\mathbf{6}$ & 4.2 & $1.13 \mathrm{E}+13$ \\
\hline TA-29 & $\mathbf{5}$ & 3.7 & $\mathbf{6}$ & 4.2 & $1.13 \mathrm{E}+13$ \\
\hline TA-30 & $\mathbf{5}$ & 3.7 & $\mathbf{6}$ & 4.2 & $2.22 \mathrm{E}+13$ \\
\hline TA-31 & $\mathbf{5}$ & 3.7 & $\mathbf{6}$ & 4.2 & $2.20 \mathrm{E}+13$ \\
\hline TA-32 & $\mathbf{2}$ & 3.5 & $\mathbf{5}$ & 3.7 & $2.28 \mathrm{E}+13$ \\
\hline TA-33 & $\mathbf{2}$ & 3.5 & $\mathbf{5}$ & 3.7 & $2.27 \mathrm{E}+13$ \\
\hline TA-34 & $\mathbf{1 5}$ & 3.7 & $\mathbf{1 6}$ & 4.0 & $2.27 \mathrm{E}+13$ \\
\hline TA-35 & $\mathbf{1 5}$ & 3.7 & $\mathbf{1 6}$ & $2.27 \mathrm{E}+13$ \\
\hline
\end{tabular}



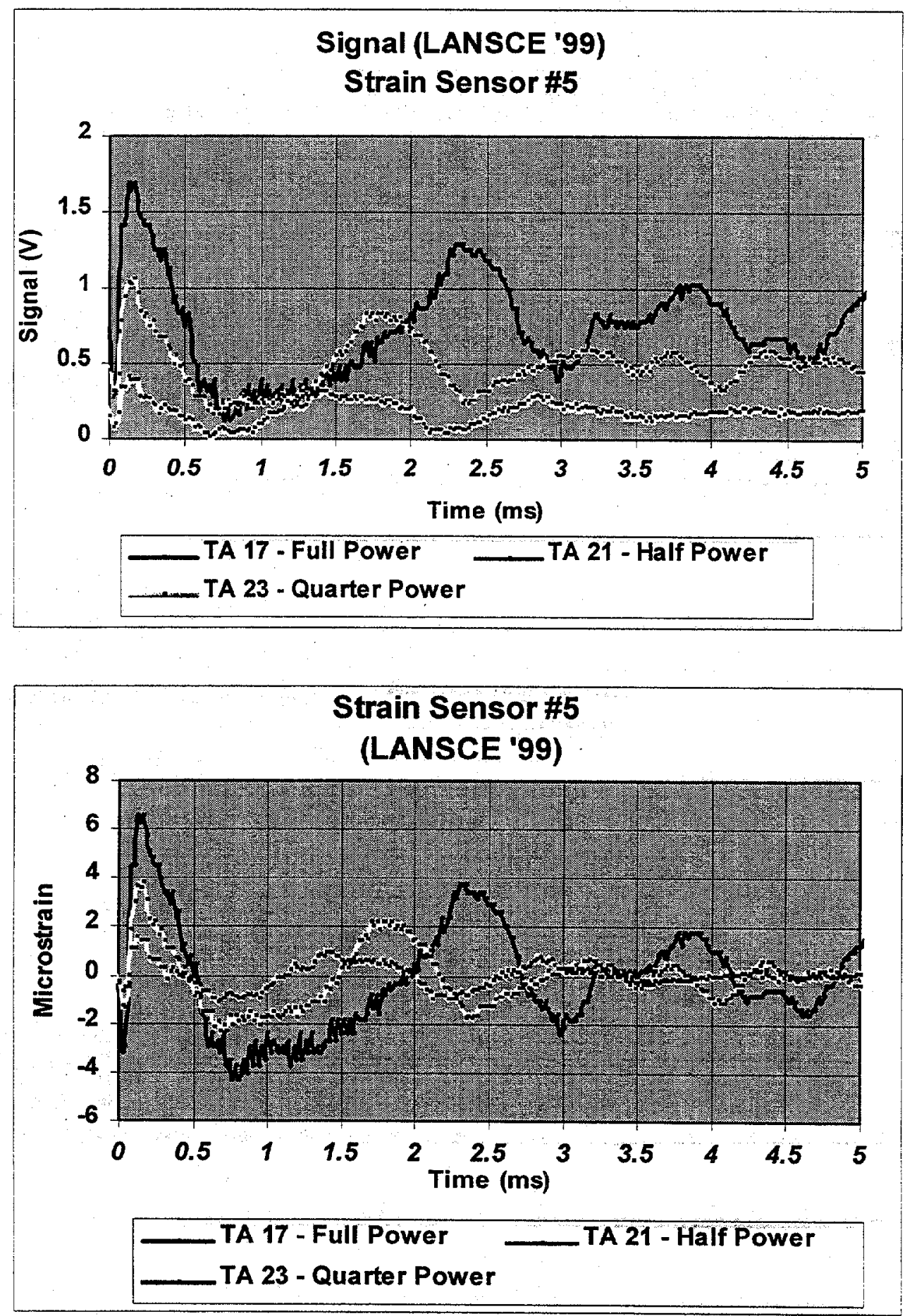

Figure 6.a. Strain sensor 5 (axial orientation) data and extracted strain for pulses TA17, 21, 23. 

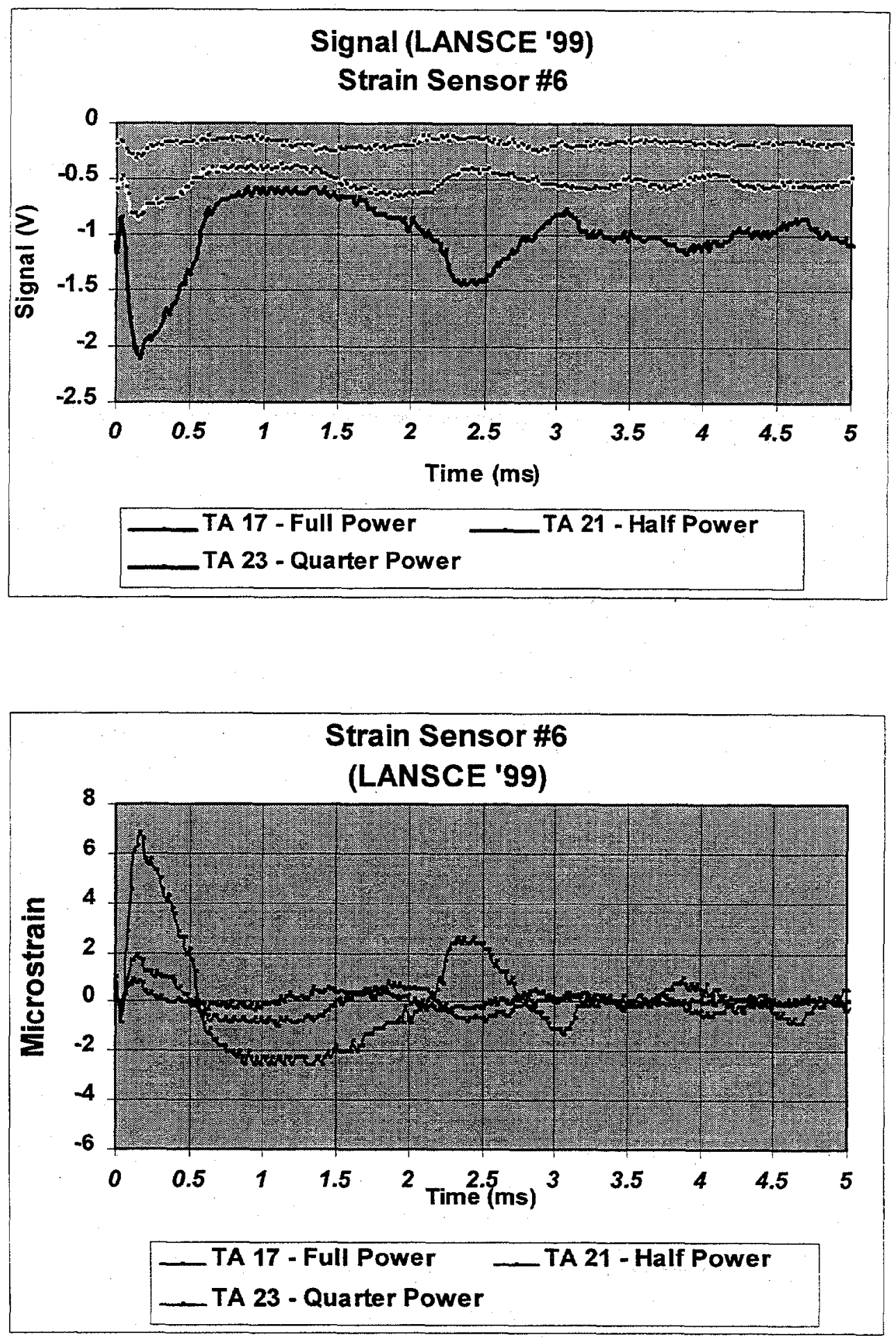

Figure 6.b. Strain sensor 6 (axial orientation) data and extracted strain for pulses TA$17,21,23$. 
Figure 7 shows the data taken from sensors 19 and 20 . (See Figure 5.) These sensors were arranged to respond to transverse (hoop) strain. Their response is indicative of the higher strain produced in that direction, compared with the axial. For these data it is necessary to follow the signal through either the maximum or minimum voltage for the sensor, or both; that is, the signal goes outside the monotonic range. The extracted strain versus time profile in the figure illustrates this departure from monotonic. The peak strain values seen are approximately an order of magnitude greater than their counterparts in the axial direction.

\section{CONCLUSIONS}

Several important general conclusions can be drawn from the experimental data. The first is that highsensitivity optical strain measurements yield reasonable data despite the high transient electromagnetic field of each proton pulse. This fact is verification of the importance of using optical sensors in this environment. In addition, the sensitivity of interferometric systems was necessary for the range of strains produced, a sensitivity not available in standard strain gauges. A related second conclusion is that this relatively fragile type of sensor can be effectively attached to the target surfaces, will survive the thermal shock, and can be connected through long optical paths to instrumentation outside the test chamber. With respect to the data signals themselves, an important general conclusion is that data are consistent with beam power scaling and tend to scale in correspondence to position and orientation on the target surface.

The data also show that axial strains (those parallel to the long axis through the target) tend to be at least an order of magnitude less than those around the cylindrical perimeter (hoop strains). The strain per unit time was measured with up to about $100 \mathrm{KHz}$ time response and tended, for most pulses, to persist above the background for $10 \mathrm{~ms}$ or more. The values of the strain for axial sensors 5 and 6 , studied for a number of pulses, are given with a high degree of certainty, both because of their consistency in pulse to pulse and their scalability with beam power. In the hoop strain measurements, e.g., for TA-9, with higher peak strains, the larger gap excursions in the sensors tended to exceed a full fringe. In these instances, some uncertainty remains as to the turnaround time of the signal, leaving the resulting peak strains somewhat more uncertain than their axial counterparts. Plans are underway for acquisition of improved measurement systems for future tests, where the problems of single wavelength measurement ambiguity are no longer at issue.

\section{ACKNOWLEDGEMENTS}

The authors would like to recognize the helpful support of Greg Chaparro and Lloyd Hunt of the Los Alamos National Laboratory and the accelerator staff at LANSCE. This work has benefited from the use of the LANSCEWNR facility that is funded by the U.S. Department of Energy under contract W-7405-ENG-36. Research at ORNL was sponsored by the Office of Energy Sciences for the U.S. Department of Energy under contract DE-AC0596OR22464 with the Lockheed Martin Research Corporation.

\section{REFERENCES}

1. J. R. Haines, T. A. Gabriel, and T. G. McManamy, "Overview of the Target Systems for the Spallation Neutron Source", Presented in the Second International Topical Meeting on Nuclear Applications of Accelerator Technology, Proceedings of AccApp 98, p. 222 (1998).

2. R. P. Taleyarkhan, S. H. Kim, and J. R. Haines, "Pretest Predictions of Surface Strain and Fluid Pressure in Mercury Targets Undergoing Thermal Shock", Transactions of the American Nuclear Society, Vol. 80, TANSAO 80, p. 311 (1999)

3. D. D. Earl, S. W. Allison, C. C. Tsai, and J. R. Haines, "Improved Fiber Optic Pressure and Strain Sensing Techniques for Use in High Radiation Environment", Presented in the Second International Topical Meeting on Nuclear Applications of Accelerator Technology, Proceedings of AccApp 98, p. 665. (1998) 

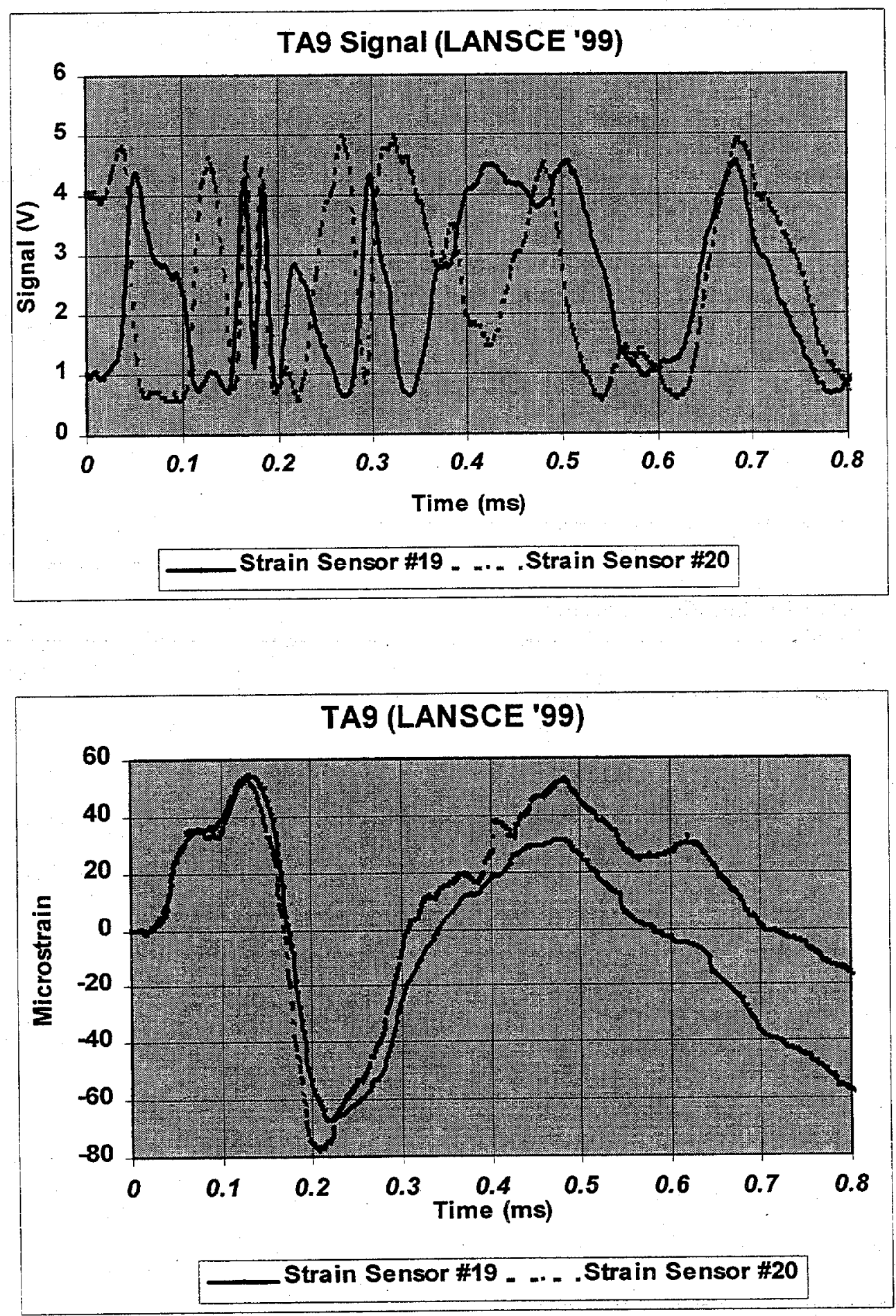

Figure 7. Strain sensors 19 and 20 (transverse orientation) data and extracted strain for pulse TA-9. 